\title{
DIAGNÓSTICOS DE ENFERMAGEM APLICADOS A PACIENTES COM INSUFICIÊNCIA CARDÍACA DESCOMPENSADA
}

\author{
Paulo Cesar da Costa Galvão ${ }^{1}$, Eduardo Tavares Gomes², Thaisa Remigio Figueirêdo ${ }^{3}$, Simone Maria Muniz \\ da Silva Bezerra ${ }^{4}$
}

\begin{abstract}
RESUMO: Objetivou-se identificar os diagnósticos de enfermagem prioritários para pacientes com insuficiência cardíaca descompensada. Trata-se de uma com abordagem quantitativa, realizada em pronto-socorro cardiológico de uma universidade de Pernambuco, entre outubro de 2014 e fevereiro de 2015. A amostra do estudo foi composta de 62 pacientes admitidos por insuficiência cardíaca descompensada, internados de emergência e de qualquer classe funcional. Os principais diagnósticos de enfermagem foram: Débito Cardíaco Diminuído (87,3\%), Intolerância à Atividade (79,4\%) e Padrão Respiratório Ineficaz (38,1\%). Os sinais e sintomas da descompensação, oriundos da congestão sistêmica ou pulmonar, serviram como base para o reconhecimento das características definidoras que permitiram a elaboração dos diagnósticos de enfermagem prioritários para esses pacientes.

DESCRITORES: Enfermagem Cardiovascular; Insuficiência Cardíaca; Diagnóstico de Enfermagem; Processos de Enfermagem.
\end{abstract}

\section{NURSING DIAGNOSIS APPLIED TO PATIENTS WITH DECOMPENSATED HEART FAILURE}

ABSTRACT: The present study aimed to identify the priority nursing diagnoses for patients with decompensated heart failure. Quantitative study conducted in a cardiac emergency service of a university of Pernambuco, between October 2014 and February 2015. The study sample was composed of 62 patients admitted for the treatment of acute decompensated heart failure, emergency inpatients and patients from any functional class. The main nursing diagnoses were Decreased Cardiac Output (87.3\%), Activity Intolerance (79.4\%) and Ineffective Breathing Pattern (38.1\%). The signs and symptoms of decompensation caused by systemic or pulmonary congestion provided the basis for the recognition of defining characteristics that made it possible to formulate priority nursing diagnoses for these patients.

DESCRIPTORS: Cardiovascular Nursing; Heart Failure; Nursing Diagnosis; Nursing Processes.

\section{DIAGNÓSTICOS DE ENFERMERÍA APLICADOS A PACIENTES CON INSUFICIENCIA CARDÍACA DESCOMPENSADA}

RESUMEN: Fue objetivo del estudio identificar los diagnósticos de enfermería prioritarios para pacientes con insuficiencia cardíaca descompensada. El abordaje fue cuantitativo y se realizó en la emergencia cardiológica de una universidad de Pernambuco, entre octubre de 2014 y febrero de 2015. La muestra del estudio fue compuesta de 62 pacientes admitidos por insuficiencia cardíaca descompensada, internados en emergencia y de cualquier clase funcional. Los principales diagnósticos de enfermería fueron: Débito Cardíaco Disminuido (87,3\%), Intolerancia a la Actividad (79,4\%) y Patrón Respiratorio Ineficaz (38,1\%). Las señales y síntomas de la descompensación, que vienen de la congestión sistémica o pulmonar, fueron utilizadas como base para el reconocimiento de las características determinantes, las cuales posibilitaron la elaboración de los diagnósticos de enfermería prioritarios para eses pacientes.

DESCRIPTORES: Enfermería Cardiovascular; Insuficiencia Cardíaca; Diagnóstico de Enfermería; Procesos de Enfermería.

${ }^{1}$ Discente de Enfermagem. Faculdade de Enfermagem Nossa Senhora das Graças. Universidade de Pernambuco. Recife, PE, Brasil.

${ }^{2}$ Enfermeiro. Mestrando em Enfermagem. Universidade de Pernambuco. Recife, PE, Brasil.

${ }^{3}$ Enfermeira. Doutoranda em Enfermagem. Universidade de Pernambuco. Recife, PE, Brasil.

${ }^{4}$ Enfermeira. Pós-doutora em Enfermagem Fundamental. Docente do Programa de Pós-Graduação Associado em Enfermagem. Universidade de Pernambuco. Recife, PE, Brasil. 


\section{INTRODUÇÃO}

A Insuficiência Cardíaca (IC) é uma síndrome crônica e progressiva, associada a readmissões hospitalares, baixa qualidade de vida, risco de mortalidade precoce e geradora de altos custos para o sistema de saúde. É uma doença de caráter sistêmico, definida como disfunção cardíaca que ocasiona inadequado suprimento sanguíneo para atender necessidades metabólicas tissulares, na presença de retorno venoso normal, ou fazê-lo somente com elevadas pressões de enchimento ${ }^{(1)}$. Evidenciada por um conjunto de sinais e sintomas de congestão pulmonar e sistêmica, é caracterizada como o último estágio de todas as doenças cardiovasculares ${ }^{(2)}$.

Essa síndrome afeta de 1,5 a 2\% da população mundial e sua incidência vem aumentando nas últimas três décadas, especialmente em indivíduos com mais de 65 anos. Apesar dos benefícios alcançados com a evolução no tratamento da IC, essa síndrome ainda é responsável por altas taxas de mortalidade e pela diminuiç̧ão da capacidade funcional de seus portadores ${ }^{(1-2)}$.

O aumento na incidência de IC está relacionado aos avanços terapêuticos no tratamento do infarto agudo do miocárdio, da hipertensão arterial e da mesma, o que ocasiona maior sobrevida e, consequentemente, aumento da prevalência e de internações hospitalares por essa síndrome, gerando altos custos para países cuja população idosa é crescente ${ }^{(3)}$.

Considerada um dos maiores problemas de saúde pública, a IC traz significativos custos para o sistema de saúde, por contribuir para o aumento das taxas de readmissões, além de altas taxas de morbidade, mortalidade e letalidade ${ }^{(4)}$. Observam-se percentuais bastante elevados dessas taxas após a primeira internação por descompensação da IC principalmente no período de 30 a 90 dias após a $\operatorname{alta}^{(1,3)}$.

Neste contexto, o papel do enfermeiro na IC tem sido fortemente focado em intervenções terapêuticas, educativas e de autocuidado. A efetividade do diagnóstico de enfermagem em pacientes em domicílio, em ambulatório e de hospitalização com insuficiência cardíaca deve ser explorada, dada sua relevância para a prática clínica.

O Processo de Enfermagem (PE), em todas as suas etapas, constitui-se como ferramenta essencial na prática clínica do enfermeiro e determina o estabelecimento do diagnóstico de enfermagem (DE), que é considerado o norteador para a escolha das intervenções mais adequadas visando alcançar os resultados esperados a cada indivíduo no contexto do cuidado. A Sistematização da Assistência de Enfermagem é atividade privativa e de responsabilidade do enfermeiro, mediante a utilização de taxonomias validadas internacionalmente para todas as etapas do processo ${ }^{(5)}$.

O treinamento e o desenvolvimento de habilidades na avaliação clínica na prática aproximam o PE da prática baseada em evidências. As taxonomias para os DE favorecem o raciocínio crítico na realização do $\mathrm{PE}$, particularmente acerca das características definidoras, tendo por referência sinais e sintomas apresentados, e a eleição dos diagnósticos prioritários ${ }^{(6)}$.

Entende-se que uma boa avaliação clínica pode identificar pacientes com quadros congestivos ou hipovolêmicos com confiabilidade razoável, bem como pacientes com baixo débito cardíaco ou normal. Estudos confirmam que os enfermeiros possuem capacidade de realizar uma avaliação clínica confiável nesses pacientes com insuficiência cardíaca, melhorando os seus resultados clínicos $^{(7)}$.

Esta pesquisa enfatiza a importância do estabelecimento dos diagnósticos de enfermagem na condução da IC descompensada estabelecendo um paralelo entre as manifestações clínicas apresentadas e o processo de enfermagem. Nessa perspectiva, este estudo tem como objetivo identificar, através do perfil sintomático do paciente, os DE prioritários para pacientes com insuficiência cardíaca descompensada.

\section{MÉTODO}

Trata-se de uma pesquisa do tipo descritiva e exploratória com abordagem quantitativa, realizada no Pronto-Socorro Cardiológico de Pernambuco Professor Luiz Tavares da Universidade de Pernambuco 
(PROCAPE/UPE) entre outubro de 2014 e fevereiro de 2015. A instituição caracteriza-se como hospital universitário de médio porte oferecendo serviços de média e alta complexidade em Cardiologia, conveniado ao Sistema Único de Saúde (SUS), com internamento e acompanhamento ambulatorial.

A amostragem foi por conveniência sendo incluídos os pacientes que atendiam aos critérios de inclusão/exclusão. A população do estudo foi composta de pacientes admitidos por IC descompensada, internados na emergência com diagnóstico de IC de qualquer classe funcional, conforme classificação de New York Heart Association (NYHA), de qualquer etiologia e com fração de ejeção $\leq 45 \%$, em internamento consequente à congestão pulmonar ou sistêmica e seus efeitos ${ }^{(1)}$. A classificação da NYHA permite estratificar o grau de limitação imposto pela IC para atividades cotidianas em função da apresentação dos sintomas. Segundo a classificação, os indivíduos com IC são classificados em quatro: classe I - ausência de sintomas durante atividades cotidianas, com limitação para esforços semelhante à esperada em indivíduos saudáveis; classe II - sintomas desencadeados por atividades cotidianas; classe III - sintomas desencadeados em atividades menos intensas que as cotidianas; classe IV - sintomas em repouso.

Para que os pacientes pudessem participar da pesquisa, foram adotados os seguintes critérios de inclusão: idade superior a 18 anos, diagnóstico médico de insuficiência cardíaca, estar no primeiro dia de internação hospitalar, possibilidade de comunicação/expressão verbal, estado neurológico/ cognitivo preservado e aceitar participar da pesquisa através da assinatura do Termo de Consentimento Livre e Esclarecido.

Foram excluídos pacientes com IC após infarto agudo do miocárdio nos três meses prévios à internação, pacientes com IC secundária a quadro de sepse, pacientes submetidos a cirurgia de revascularização do miocárdio nos 30 dias prévios à internação e pacientes com sequelas cognitivas.

Dados referentes à identificação, variáveis demográficas e clínicas relativas à classe funcional e etiologia da IC, bem como principais sinais e sintomas na admissão, foram coletados mediante entrevista (anamnese), exame físico e consulta ao prontuário clínico, com vistas a inferir os DE prioritários aos pacientes. Não foram consultados dados de internações anteriores.

O raciocínio clínico e julgamento diagnóstico para inferir os $\mathrm{DE}$, a partir dos sinais e sintomas identificados, foi realizado com base na utilização de linguagem padronizada da Taxonomia II da North American Nursing Diagnoses Association (NANDA)(8). O instrumento de coleta foi elaborado contendo 33 DE possíveis, mais a possibilidade de registro de outros. Foram incluídos DE dos domínios: 2 - Nutrição; 3 - Eliminação e troca; 4 - Atividade/Repouso; 9 - Enfrentamento/Tolerância ao estresse; 11 - Segurança/Proteção ${ }^{(7-8)}$.

As variáveis foram tabuladas e analisadas pelo software Statistical Package for the Social Sciences (SPSS), versão 20.0, a partir de estatística descritiva.

O presente estudo foi previamente aprovado (maio de 2013) pelo Comitê de Ética em Pesquisa sob parecer n. 255.064, CAAE: 03198212.1.0000.5201.

\section{- RESULTADOS}

Foram realizadas 62 entrevistas dentre as quais $61,3 \%$ dos indivíduos eram do sexo masculino, $56,5 \%$ declaravam-se pardos e $54,84 \%$ tinham idade superior a 60 anos. A maioria era casada ou em união estável $(48,4 \%)$, residia na zona urbana $(82,3 \%)$, trabalhava como autônomos $(41,9 \%)$ ou eram aposentados (32,3\%), conforme Tabela 1.

Os principais fatores precipitantes do último episódio de descompensação da insuficiência cardíaca foram a interrupção de medicação $(27,4 \%)$, arritmias $(24,4 \%)$, ingestão hídrica ou salina excessiva $(17,7 \%)$, e crise hipertensiva $(17,7 \%)$. As principais causas levantadas como predisponentes do diagnóstico médico de IC foram Cardiomiopatia (35,5\%), Doença de Chagas (27,4\%) e Hipertensão arterial (9,7\%). Dentre os entrevistados, identificou-se $41,7 \%$ em classe funcional III e 35,5\% em classe funcional IV (Tabela 2).

Dentre os fatores de risco para IC investigados, destacaram-se hipertensão arterial (66,1\%), etilismo 
$(61,3 \%)$, histórico familiar de IC (56,5\%), tabagismo (54,8\%), diabetes $(32,3 \%)$ e dislipidemia $(17,7 \%)$. Durante o exame físico, os principais sinais e sintomas encontrados foram cansaço $(77,4 \%)$, dispneia paroxística noturna $(69,4 \%)$ e dispneia $(48,4 \%)$, de acordo com a Tabela 2.

Após a avaliação dos pacientes, os principais DE elencados são apresentados na Tabela 3, com as respectivas frequências e fatores relacionados, considerando a possibilidade de múltiplos diagnósticos por paciente.

Tabela 1 - Caracterização sociodemográfica dos pacientes com insuficiência cardíaca. Recife, PE, Brasil, 2015

\begin{tabular}{lcc} 
Variáveis & N & \% \\
\hline Masculino & 38 & 61,3 \\
\hline Feminino & 24 & 38,7 \\
\hline Até 60 anos & 28 & 45,16 \\
\hline Mais de 60 anos & 34 & 54,84 \\
\hline Casado/ União estável & 30 & 48,4 \\
\hline Solteiro & 19 & 30,6 \\
\hline Viúvo & 10 & 16,1 \\
\hline Separado/ Divorciado & 1 & 1,6 \\
\hline Outro & 2 & 3,2 \\
\hline Branco & 12 & 19,4 \\
\hline Negro(a) & 13 & 21 \\
\hline Pardo(a) & 35 & 56,5 \\
\hline Amarelo(a) & 2 & 3,2 \\
\hline Zona Urbana & 51 & 82,3 \\
\hline Zona Rural & 11 & 17,7 \\
\hline Aposentado & 20 & 32,3 \\
\hline Assalariado & 12 & 19,4 \\
\hline Autônomo & 26 & 41,9 \\
\hline Sem renda fixa & 4 & 6,5
\end{tabular}

Tabela 2 - Distribuição dos pacientes em variáveis relacionadas à insuficiência cardíaca. Recife, PE, Brasil, 2015

\begin{tabular}{|c|c|c|}
\hline Variáveis & $\mathbf{N}$ & $\%$ \\
\hline \multicolumn{3}{|c|}{ Fatores precipitantes da descompensação } \\
\hline Interrupção de Medicação & 17 & 27,4 \\
\hline Arritmias & 15 & 24,4 \\
\hline Ingestão hídrica ou salina excessiva & 11 & 17,7 \\
\hline Hipertensão Arterial & 11 & 17,7 \\
\hline Álcool & 7 & 11,3 \\
\hline Insuficiência Renal & 6 & 9,7 \\
\hline Infecção & 4 & 6,5 \\
\hline Medicamentos & 3 & 4,8 \\
\hline Anemia & 1 & 1,8 \\
\hline Hospitalização prévia & 46 & 74,2 \\
\hline \multicolumn{3}{|l|}{ Etiologia da Insuficiência Cardíaca } \\
\hline Cardiomiopatia & 22 & 35,5 \\
\hline Doença de Chagas & 17 & 27,4 \\
\hline Outras & 10 & 16,1 \\
\hline Hipertensão arterial & 6 & 9,7 \\
\hline Doença isquêmica & 1 & 1,6 \\
\hline \multicolumn{3}{|l|}{ Classe Funcional } \\
\hline $\mathrm{I}$ & 6 & 9,7 \\
\hline II & 9 & 15 \\
\hline III & 25 & 41,7 \\
\hline IV & 22 & 35,5 \\
\hline \multicolumn{3}{|l|}{ Fatores de risco para IC } \\
\hline Hipertensão arterial & 41 & 66,1 \\
\hline Etilismo & 38 & 61,3 \\
\hline História familiar de IC & 35 & 56,5 \\
\hline Tabagismo & 34 & 54,8 \\
\hline Diabetes & 20 & 32,3 \\
\hline Dislipidemia & 11 & 17,7 \\
\hline \multicolumn{3}{|l|}{ Sinais e sintomas ao exame físico } \\
\hline Edema & 49 & 79 \\
\hline Cansaço & 48 & 77,4 \\
\hline Dispnéia paroxística noturna & 43 & 69,4 \\
\hline Dispnéia & 30 & 48,4 \\
\hline Dor torácica & 12 & 19,4 \\
\hline Ortopneia & 5 & 8,1 \\
\hline Palpitação & 4 & 6,5 \\
\hline Outros - inespecíficos & 20 & 32,2 \\
\hline
\end{tabular}


Tabela 3 - Diagnósticos de enfermagem e respectivos fatores relacionados aplicados à pacientes com insuficiência cardíaca. Recife, PE, Brasil, 2015

\begin{tabular}{|c|c|c|c|}
\hline Diagnóstico de Enfermagem (n(\%)) & Fator Relacionado & $\mathbf{N}$ & $\%$ \\
\hline \multirow{4}{*}{$\begin{array}{l}\text { (00029) Débito Cardíaco Diminuído } \\
55(87,3 \%)\end{array}$} & Volume de ejeção Alterado & 40 & 72,7 \\
\hline & Frequência Cardíaca Alterada & 22 & 40 \\
\hline & Ritmo Alterado & 5 & 9,1 \\
\hline & Contratilidade Alterada & 2 & 3,2 \\
\hline \multirow{3}{*}{$\begin{array}{l}\text { (00026) Volume de Líquidos Excessivo } \\
\qquad 18(28,6 \%)\end{array}$} & Mecanismos reguladores comprometidos & 12 & 66,7 \\
\hline & Ingesta Excessiva de líquidos & 2 & 11,1 \\
\hline & Ingesta Excessiva de sódio & 2 & 11,1 \\
\hline \multirow{5}{*}{$\begin{array}{c}\text { (00032) Padrão Respiratório Ineficaz } \\
24(38,1 \%)\end{array}$} & Hiperventilação & 22 & 91,7 \\
\hline & Fadiga da musculatura Respiratória & 8 & 33,3 \\
\hline & Dor & 6 & 25 \\
\hline & Obesidade & 3 & 12,5 \\
\hline & Fatores Metabólicos & 1 & 4,2 \\
\hline \multirow[t]{2}{*}{$\begin{array}{l}\text { (00092) Intolerância à atividade } \\
50(79,4 \%)\end{array}$} & $\begin{array}{l}\text { Desequilíbrio entre a oferta e a demanda } \\
\text { de oxigênio }\end{array}$ & 49 & 98 \\
\hline & Fraqueza generalizada & 1 & 2 \\
\hline \multirow{5}{*}{$\begin{array}{l}\text { (00085) Mobilidade física prejudicada } \\
30(47,6 \%)\end{array}$} & Intolerância à atividade & 26 & 86,7 \\
\hline & Desconforto & 6 & 20 \\
\hline & Controle muscular diminuído & 5 & 16,7 \\
\hline & Ansiedade & 2 & 6,7 \\
\hline & Dor & 2 & 6,7 \\
\hline \multirow{5}{*}{$\begin{array}{l}\text { (00046) Integridade da pele prejudicada } \\
14(22,2 \%)\end{array}$} & Circulação prejudicada & 13 & 92,9 \\
\hline & Mudanças no turgor & 2 & 14,3 \\
\hline & Hipertermia & 1 & 7,1 \\
\hline & Hipotermia & 1 & 7,1 \\
\hline & Estado metabólico prejudicado & 1 & 7,1 \\
\hline $\begin{array}{l}\text { (00033) Ventilação Espontânea Prejudicada } \\
9(14,3 \%)\end{array}$ & Ansiedade & 2 & 8,3 \\
\hline \multirow{2}{*}{$\begin{array}{l}\text { (00146) Ansiedade } \\
2(3,2 \%)\end{array}$} & Ameaça de morte & 1 & 50 \\
\hline & Estresse & 1 & 50 \\
\hline
\end{tabular}

\section{- DISCUSSÃO}

Os pacientes avaliados apresentaram perfil sociodemográfico e clínico similar a de outros estudos nacionais ${ }^{(9-10)}$. Com uma amostra similar, um estudo nacional demonstrou que o diagnóstico de IC é desconhecido por $75,7 \%$ dos pacientes e $40,5 \%$ procuram os serviços de saúde apenas em casos de urgência $^{(9)}$.

O enfermeiro tem papel fundamental no manejo do paciente descompensado por insuficiência cardíaca, sendo o responsável principal pela orientação terapêutica visando à adesão medicamentosa ou não-farmacológica. Uma pesquisa nacional revelou que para os enfermeiros, a principal causa da não realização das suas atividades educativas é a falta de tempo $(87 \%)$, sugerindo que essa atividade, primordial, é consumida pelas demais no processo de trabalho do enfermeiro ${ }^{(11)}$.

Foram elencados oito DE prioritários para os pacientes avaliados. Uma revisão da literatura mostrou que, além dos diagnósticos ora apresentados, Risco de queda e Conhecimento deficiente também estavam entre os mais encontrados ${ }^{(12)}$. 
Em pesquisa realizada com pacientes cardiopatas hospitalizados, incluindo portadores de IC e de infarto, os principais DE aplicados foram: Ansiedade (76,7\%), Dor Aguda (70,7\%), Débito Cardíaco Diminuído (56,7\%), Percepção Sensorial Perturbada - Visual (53,3\%), Insônia (46,7\%), Intolerância à Atividade (36,7\%), Disfunção Sexual (36,7\%) e Eliminação Urinária Prejudicada $(36,7 \%)$, sendo a ansiedade e a dor mais frequentes em pacientes com infarto ${ }^{(13)}$.

Em outro estudo nacional, com levantamento dos DE pela inferência a partir dos sinais e sintomas apresentados, os autores encontraram três DE considerados como prioritários para uma amostra de 303 pacientes com IC, e também encontrados em nossos achados: Débito Cardíaco Diminuído, Volume de Líquidos Excessivo, Padrão Respiratório Ineficaz ${ }^{(6)}$.

O Débito Cardíaco Diminuído, encontrado em 87,3\% dos pesquisados, está relacionado principalmente à diminuição da fração de ejeção do ventrículo esquerdo. É um DE validado em português e com definições operacionais objetivas para todas as características definidoras, que se correlacionam com sinais e sintomas ${ }^{(6,14-16)}$. Um estudo retrospectivo de 38 pacientes com IC em avaliação para transplante verificou que $71,1 \%$ apresentavam esse $\mathrm{DE}$, sendo que as maiores razões de chance foram a resistência vascular sistêmica aumentada $(O R=4,53)$, a terceira bulha $(O R=3,429)$, e a fração de ejeção diminuída $(\mathrm{OR}=2,85)^{(17)}$.

O padrão respiratório ineficaz tem alta prevalência entre pacientes com IC, levando a prejuízo do sono. Uma pesquisa com 400 pacientes em Classe Funcional II e III com prejuízo do sono mostrou que o desconforto respiratório estava presente em 40,5\% da amostra, perdendo para a nictúria $(72,2 \%)$, acordar no meio da noite ou de manhã cedo $(67,7 \%)$, dificuldade para dormir em até 30 minutos $(56,5 \%)$. Os autores sugerem que a nictúria pode estar relacionada ao Volume Excessivo de Líquidos ou ao uso inadequado dos diuréticos ${ }^{(18)}$. Nos achados ora apresentados, o DE Volume Excessivo de Líquidos foi aplicado a $28,6 \%$ dos pacientes, dos quais $66,7 \%$ tinham por fator relacionado mecanismos reguladores comprometidos, podendo ser por influência do uso inadequado de diuréticos.

Em uma pesquisa com amostra com 37 pacientes com IC internados em um hospital com objetivo de avaliar o grau de comprometimento da troca gasosa nesses pacientes, os indicadores clínicos profundidade e frequência respiratória, ortopneia, dores no peito, sonolência e ureia foram os mais comprometidos. Para os autores, os pacientes ou apresentavam o DE ou o risco para o mesmo ${ }^{(19)}$.

A Intolerância à Atividade tem por característica definidora o relato de fadiga, muito frequente em pacientes com $\mathrm{IC}^{(6,8)}$. Outro estudo de revisão aponta que fadiga na $\mathrm{IC}$ está relacionada à baixa perfusão muscular e geralmente é acompanhada por dispneia e se associa a variáveis psicossociais. Nas pesquisas revisadas, a incidência foi de $69 \%$ a $88 \%{ }^{(20)}$, sendo encontrado neste trabalho de $79,4 \%$. O principal fator relacionado a esse $\mathrm{DE}$, para a maioria dos pacientes desta amostra (98\%), foi o desequilíbrio entre a oferta e a demanda de oxigênio, que deve estar associado ao baixo débito com conseguinte diminuição da oferta de oxigênio aos tecidos.

Por fim, vale ressaltar que o enfermeiro como educador tem compromisso com o manejo da IC na atenção primária para minimizar complicações e melhorar qualidade de vida. Em outras publicações, ficou evidente elevada presença do diagnóstico de enfermagem Conhecimento Deficiente ${ }^{(12)}$. Muitos desses pacientes hospitalizados descobrem-se em cuidados paliativos, necessitando ainda mais de educação, monitoramento, mudança no estilo de vida e cuidados profissionais ${ }^{(1)}$.

A educação em saúde para esses pacientes tem dado enfoque ao conhecimento da doença, monitorização dos sinais e sintomas de descompensação, uso de medicamentos e aderência a medidas não-farmacológicas ${ }^{(21)}$. Esses tópicos devem ser abordados ainda no internamento, devendo fazer parte do plano de alta hospitalar e seguindo no acompanhamento ambulatorial.

Em uma investigação realizada mediante visitas domiciliares a pacientes com IC, ficou demonstrado que os pacientes tinham menor conhecimento das questões relacionadas às drogas envolvidas no tratamento e aos efeitos colaterais. Essa pesquisa avaliou um incremento significativo no conhecimento dos pacientes numa segunda visita após uma intervenção educativa inicial, sugerindo que ações como essa na atenção básica podem impactar na adesão terapêutica e diminuir hospitalizações ${ }^{(22)}$. Outro estudo, mais robusto, realizado na Colômbia, com um grupo controle, sem cegamento, avaliou que um protocolo educativo de enfermagem para pacientes com IC foi significativo no aumento de no mínimo $20 \%$ no escore de autocuidado em $66 \%$ do grupo intervenção, contra $26,6 \%$ do grupo controle ${ }^{(23)}$. 
Os pacientes com insuficiência cardíaca possuem demandas específicas de cuidado. Os sinais e sintomas da descompensação, oriundos da congestão sistêmica ou pulmonar, foram a base para o reconhecimento das características definidoras que permitiram a elaboração dos DE prioritários.

Os indivíduos investigados apresentaram perfil de diagnósticos similar a outros estudos. Sugere-se ainda investigar diagnósticos de outros domínios da taxonomia NANDA II, ainda não considerados, como os dos domínios 7 - Papéis e relacionamentos, 8 - Sexualidade, 10 - Princípios de Vida e 12 Conforto. Esses domínios são compostos por DE que estão relacionados a formas de enfrentamento e de adaptação a doença. Os domínios investigados abordam necessidades humanas básicas, relacionados a aspectos fisiológicos, enquanto que a investigação destes outros domínios possibilitará a compreensão holística do paciente nessas condições.

O presente estudo serve de referência para os enfermeiros que venham a lidar com este perfil de pacientes, auxiliando a reflexão que possa aprimorar a prática e a aplicação do PE.

Por fim, vale reforçar a conscientização do enfermeiro assistencial do seu papel como educador, na atenção básica ou no internamento, favorecendo a melhora da qualidade de vida, adesão terapêutica e evitando novas descompensações.

\section{REFERÊNCIAS}

1. Bocchi EA, Marcondes-Braga FG, Bacal F, Ferraz AS, Albuquerque D, Rodrigues D, et al. Sociedade Brasileira de Cardiologia. Atualização da Diretriz Brasileira de Insuficiência Cardíaca Crônica - 2012. Arq Bras Cardiol. 2012: 98(1 supl. 1): 1-33.

2. Linhares JC, Aliti GB, Castro RA, Rabelo ER. Prescrição e realização do manejo não farmacológico para pacientes com insuficiência cardíaca descompensada. Rev latino Am enferm [Internet]. 2010; 18(6) [acesso em 07 jan 2016]. Disponível: http://dx.doi.org/10.1590/S0104-11692010000600015

3. Nogueira PR, Rassi S, Correa KS. Perfil epidemiológico, clínico e terapêutico da insuficiência cardíaca em hospital terciário. Arq Bras Cardiol. 2010; 95(3): 392-398.

4. Gaui EM, Klein CH, Oliveira GMM. Mortalidade por Insuficiência Cardíaca: Análise Ampliada e Tendência Temporal em Três Estados do Brasil. Arq Bras Cardiol 2010; 94(1): 55-61.

5. Conselho Federal de Enfermagem. Resolução n. 358, de 15 outubro 2009. Dispõe sobre a Sistematização da Assistência de Enfermagem e a implementação do Processo de Enfermagem em ambientes, públicos ou privados, em que ocorre o cuidado profissional de Enfermagem. Rio de Janeiro: COFEN; 2009.

6. Aliti GB, Linhares JCC, Linch GFC, Ruschel KB, Rabelo ER. Sinais e sintomas de pacientes com insuficiência cardíaca descompensada: inferência dos diagnósticos de enfermagem prioritários. Rev gaúch enferm [Internet]. 2011;32(3) [acesso em 07 jan 2016]. Disponível: http://dx.doi.org/10.1590/S1983-14472011000300022

7. Sauer J, Rabelo ER, Castro RA, Goldraich L, RohdeLE, Clausell N, et al. Nurses' performance in classifying heart failure patients based on physical exam: comparison with cardiologist's physical exam and levels of $\mathrm{N}$-terminal pro-B-type natriuretic peptide. J Clin Nurs. [Internet] 2010; 19(23/24) [acesso em 07 jan 2016]. Disponível: dx.doi. org/10.1111/j.1365-2702.2010.03403.x

8. North American Nursing Diagnosis Association (NANDA). Diagnósticos de Enfermagem da NANDA: definições e classificação 2012/2014. Porto Alegre: Artmed; 2013.

9. Almeida GAS, Teixeira JBA, Barichello E, Barbosa MH. Perfil de saúde de pacientes acometidos por insuficiência cardíaca. Esc Anna Nery. [Internet] 2013; 17(2) [acesso em 07 jan 2016]. Disponível: http://dx.doi.org/10.1590/ S1414-81452013000200018

10. Araújo AA, Sousa MM de, Silva EP, Santos SR, Costa MML, Silva Filho IG. Clinical and epidemiological profile of patients suffering from heart failure. Rev enferm UFPE online. [Internet] 2014; 8(3) [acesso em 07 jan 2016]. 
11. Machado CGD, Wansing GB, Moraes MAP, Rabelo-Silva ER. Conhecimento do enfermeiro sobre insuficiência cardíaca em hospital geral. Rev enferm UFSM. [Internet] 2014; 4(4) [acesso em 07 jan 2016]. Disponível: http:// cascavel.ufsm.br/revistas/ojs-2.2.2/index.php/reufsm/article/view/11633

12. Cavalcanti ACD, Pereira JMV. Diagnósticos de enfermagem de pacientes com insuficiência cardíaca: revisão integrativa. Online braz j nurs. [Internet] 2014; 13(1) [acesso em 07 jan 2016]. Disponível: http://www.objnursing. uff.br/index.php/nursing/article/view/3916.

13. Pereira JMV, Cavalcanti ACD, Santana RF, Cassiano KM, Queluci GC, Guimarães TCF. Nursing diagnoses for inpatients with cardiovascular diseases. Esc Anna Nery. [Internet] 2011; 15(4) [acesso em 07 jan 2016]. Disponível: http://dx.doi.org/10.1590/S1414-81452011000400012

14. Brandão SMG, Altino DM, Silva RCG, Lopes JR. Defining characteristics of decreased cardiac output: A literature review. Int J Nurs Terminol Classif. 2011: 22(2): 92-102.

15. Martins QCS, Meireles PF, Rabelo ER, Aliti GB. Definições conceituais e operacionais das características definidoras do diagnóstico de enfermagem débito cardíaco diminuído. Rev enferm UFSM. [Internet] 2012; 2(2) [acesso em 07 jan 2016]. Disponível: http://cascavel.ufsm.br/revistas/ojs-2.2.2/index.php/reufsm/article/ view/3191/3770

16. de Souza V, Zeitoun SS, de Barros ALBL. Débito cardíaco diminuído: revisão sistemática das características definidoras. Acta paul enferm. [Internet] 2011; 24(1) [acesso em 07 jan 2016]. Disponivel: http://dx.doi.org/10.1590/ S0103-21002011000100017

17. Matos LN, Guimarães TCF, Brandão MAG, Santoro DC. Prevalence of nursing diagnosis of decreased cardiac output and the predictive value of defining characteristics in patients under evaluation for heart transplant. Rev latino am enferm. [Internet] 2012; 20(2) [acesso em 07 jan 2016]. Disponível: http://dx.doi.org/10.1590/S010411692012000200013

18. dos Santos MA, Guedes ES, Barbosa RL, da Cruz DALM. Dificuldades do sono relatadas por pacientes com insuficiência cardíaca. Rev latinoam enferm. [Internet] 2012; 20(4) [acesso em 07 jan 2016]. Disponível: http:// dx.doi.org/10.1590/S0104-11692012000400003

19. Souza VEC, Montoril MH, Pascoal LM, Lopes MVO. Avaliação da troca gasosa de pacientes com insuficiência cardíaca congestiva. Cogitare enferm. [Internet] 2010; 15(4) [acesso em 07 jan 2016]. Disponível: http://dx.doi. org/10.5380/ce.v15i4.20365

20. Fini A, da Cruz DALM. Características da fadiga de pacientes com insuficiência cardíaca: revisão de literatura. Rev latinoam enferm. [Internet] 2009; 17(4) [acesso em 07 jan 2016]. Disponível: http://dx.doi.org/10.1590/S010411692009000400019

21. Gonçalves FG, Albuquerque DC. Educação em saúde de pacientes portadores de insuficiência cardíaca. Rev enferm UERJ. [Internet] 2014; 22(3): 422-8 [acesso em 07 jan 2016]. Disponível: http://dx.doi.org/10.9789/21755361.2010.v0i0.\%25p

22. Bertuzzi D, de Souza EN, Moraes MA, Mussi C, Rabelo ER. The knowledge of patients with heart failure in the homecare context: an experimental study. Online braz j nurs. [Internet] 2012; 11(3) [acesso em 07 jan 2016]. Disponível: http://dx.doi.org/10.5935/1676-4285.20120037

23. Rodríguez-Gázquez MA, Arredondo-Holguín E, Herrera-Cortés R. Efetividade de um programa educativo em enfermagem no autocuidado em pacientes com insuficiência cardíaca: ensaio clínico randomizado. Rev latino am. enferm. [Internet] 2012; 20(2) [acesso em 07 jan 2016]. Disponível: http://dx.doi.org/10.1590/S010411692012000200012 